\title{
SOME MECHANICAL PROPERTIES OF 10/0 MONOFILAMENT NYLON SUTURES
}

\author{
L. RENNIE, ${ }^{\prime}$ W. FLEMING, ${ }^{1}$ D. CLARK, ${ }^{2}$ C. ELLERTON ${ }^{3}$ and R. BOSANQUET ${ }^{3}$ \\ Newcastle upon Tyne
}

\begin{abstract}
SUMMARY
A short pilot study is described which investigates some of the mechanical properties of ophthalmic sutures. The Alcon 10/0 monofilament nylon suture was found to behave in a mechanically consistent manner. Like all nylon it possesses the property of 'creep', which helps to compensate for overtightening of the section during surgery.
\end{abstract}

Suture-induced corneal astigmatism is still a major cause of delay in visual recovery following corneal grafts and cataract extraction through a corneal incision, sometimes delaying a stable refractive correction for several months post-operatively. Phacoemulsification through a small posteriorly placed scleral incision results in low levels of astigmatism with early visual recovery but is not appropriate for all cases or available to all surgeons. It is well established that post-operative corneal curvature is affected by the size, position and configuration of the surgical incision, by different suture materials ${ }^{1}$ and variation in suturing technique. ${ }^{2.3}$ However, it is a common experience that despite every effort to standardise these variables the individual surgeon still faces unexpected variation in post-operative astigmatism. This study was set up to investigate whether there might be any mechanical properties of the sutures themselves which could be contributing to the variability of corneal curvatures following corneal surgery.

\section{BACKGROUND}

Ophthalmic sutures will, like all materials, deform when loaded. In surgery any stretching of the suture is likely to be relevant to the visual result. In this study the terms 'stress' and 'strain' are used. These are defined as follows:

From 'Department of Mechanical Engineering, University of Northumbria at Newcastle, Newcastle upon Tyne: 'Regional Medical Physics Department, Newcastle General Hospital, Newcastle upon Tyne; '3epartment of Ophthalmology, Newcastle General Hospital, Newcastle upon Tyne, UK.

Correspondence to: D. Clark, Regional Medical Physics Department, Newcastle General Hospital, Newcastle upon Tyne NE4 6BE, UK.

$$
\begin{gathered}
\text { Stress }=\frac{\text { load }}{\text { area }} \\
\text { Strain }=\frac{\text { extension }}{\text { original length }}
\end{gathered}
$$

In practice it may be assumed that the area of the crosssection of the suture remains constant and that stress is therefore directly proportional to load. As the stress on the material is increased the strain also increases. By plotting stress against strain a stress/strain curve can be drawn. The shape of this curve is characteristic of the particular material being studied. ${ }^{+}$An example for a traditional engineering material (1144 steel) is shown in Fig. 1. It will be seen that the slope of the curve changes after the yield point is reached, which may be relevant to ophthalmic microsurgery since the degree of stress depends upon the tightness of the suture when the surgical knot is tied.

A commonly used suture material for corneal wounds is nylon which, like many other polymers, can be subject to an additional deformation known as creep, ${ }^{5}$ i.e. the stress on a material, instead of remaining constant for a fixed extension, slowly reduces as its structure modifies with time. In the surgical situation the tension within the wound will reduce as the suture stretches. Any variation in the amount of creep between individual stitches may affect the final corneal curvature.

\section{PROCEDURE}

A test rig was constructed that enabled sutures to be loaded with a gradually increasing force and the resultant extension in their length to be measured. The forces were measured with Port-o-Gram OHAUS electronic scales with an accuracy of $\pm 0.1 \mathrm{~g}$ and the extension was measured with a Mitutoyo Digimatic Indicator with an accuracy of $\pm 0.001 \mathrm{~mm}$. In all cases the sutures assessed were the Alcon 10/0 MFN sutures typically used in our routine cataract and penetrating keratoplasty procedures. Samples from different packs with different dates and batch numbers were used. All tests were carried out in air at a temperature of $22^{\circ} \mathrm{C}$. No direct contact was made with the manufacturers. Two areas were addressed:

Eye (1994) 8, 343-345 C 1994 Royal College of Ophthalmologists 


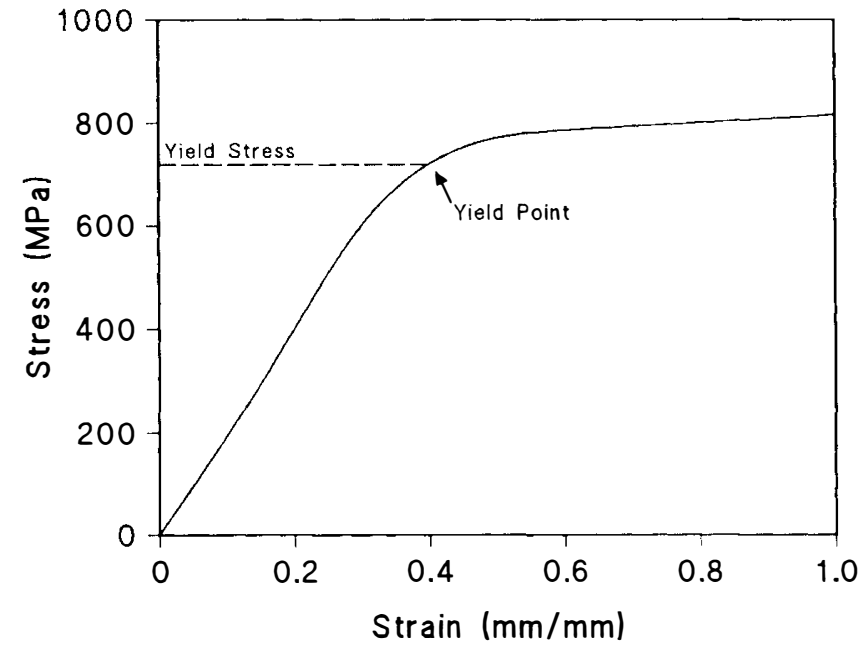

Fig. 1. Traditional material stress/strain curve.

\section{Stress/Strain Investigation}

The variability of the mechanical properties between samples of the same type of suture was investigated by comparing stress/strain curves produced from different sutures.

In order to assess the repeatability of the mechanical properties, load/extension tests were carried out for 10 sutures from separate packs, each suture being subject to increasing loads until failure occurred. Since the suture was loaded until failure and never unloaded, any effects due to hysteresis were avoided. The loading rate was maintained so as to produce an extension of $1 \mathrm{~mm}$ per 20 seconds and the load and extension were monitored over the whole test. All sutures used were taken directly from their sterile packs.

\section{Creep Deformity Investigation}

The second area investigated was that of the creep behaviour of sutures. Stitches in the eye will not all be pulled to exactly the same tension. Local oedema will also occur to different degrees. Both of these will result in the sutures

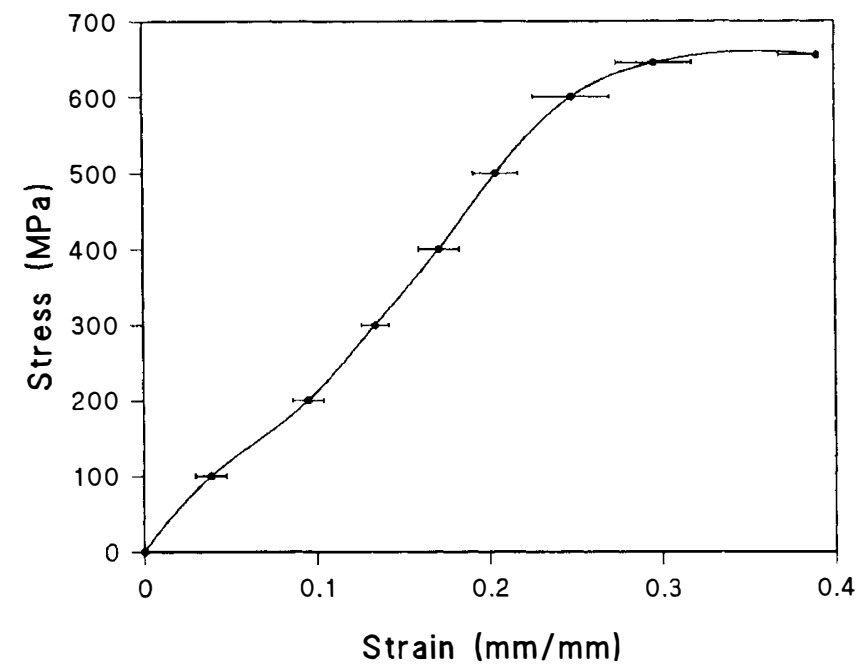

Fig. 2. Average suture stress/strain curve. Points and bars show the mean and $S D$ respectively of 10 sutures. undergoing various amounts of extension. If the creep behaviour is different for different extensions then this may lead to unpredictable and inconsistent results.

To examine the creep behaviour, a suture was given a fixed extension and the load monitored over a period of time (up to 12 hours). This was repeated for a range of extensions. Again all sutures used were taken directly from their sterile packs.

\section{RESULTS}

The results from the stress/strain investigation indicate that the sutures fail at a load of $650 \pm 15 \mathrm{MPa}$ (megapascals) and yield at a load of $570 \pm 20 \mathrm{MPa}$. Fig. 2 shows the results of the stress/strain repeatability tests. The mean of the 10 sutures is plotted with an error of \pm 1 standard deviation. The narrow spread of results (less than $\pm 3 \%$ of stress) indicates that for the stresses and strains encountered within these tests the ophthalmic suture behaved as a mechanically consistent material. It will be noted that in the early part of the test, up to the yield point, the curve is not a straight line as would be expected for a traditional engineering material. The simple explanation for this is that the suture 'relaxes' over a period of time and as these particular tests took between 300 and 1500 seconds to perform, this process was in action during the test. This could be a factor when trying to obtain consistent stitch tightness.

Fig. 3 is a summary of the results from the creep deformity investigation. In this test the suture was loaded until it reached a predetermined extension. The load induced in the suture was subsequently monitored and found to decrease with time. Moreover it can be seen from Fig. 3 that the greater the initial force on the suture, the faster this force reduces. This has a beneficial effect for the ophthalmic surgeon in that a stitch which is inserted too tightly will tend to relax more quickly than one which is at the correct tension, thereby helping to achieve a uniform surgical result.

\section{DISCUSSION}

The creep behaviour of bulk nylon is well documented,

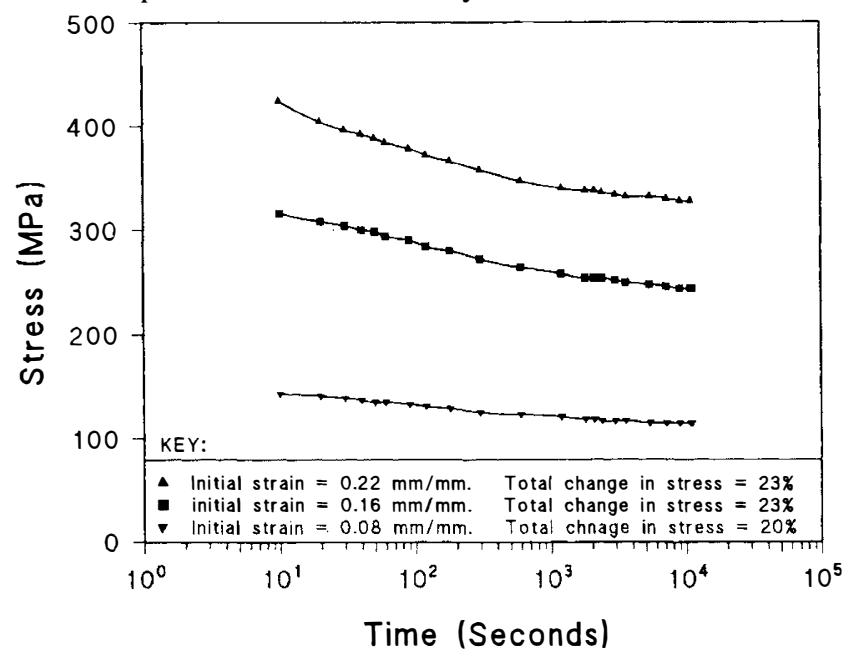

Fig. 3. Stress/time curves for sutures with different initial loadings. 
but no work has appeared in the literature on the effect which this might have in microsurgery. Also, although the uniformity of manufactured samples of bulk nylon has been studied there is little information on how this is affected by the production of small-diameter ophthalmic suture material. Kronenthal ${ }^{6}$ reported on the breaking stress of various sizes of nylon sutures from several manufacturing companies. He found large differences in suture diameter between different makes in the 10/0 nylon group 'accounting for much of the strength differences' found. Other studies have reported loss of tensile strength postoperatively (but usually of large-diameter sutures). Hutz and Ullerich ${ }^{7}$ investigated some of the physical properties of ophthalmic suture material including polyamide 6 , polyamide $6 / 6$, polyethylene terephtalate and polypropylene. The determination of short-term and long-term elasticity were reported together with the breaking stress for natural as well as synthetic fibres. Some of this work was carried out on a larger-diameter suture material and it did not look at creep per se.

Our pilot study was devised to assess the stress/strain repeatability and creep deformity of the commonly used $10 / 0$ nylon suture. Our findings have shown that the Alcon $10 / 0$ suture is a mechanically consistent material in terms of its stress/strain characteristics and therefore variations between one suture and the next cannot be the cause of variable amounts of post-operative astigmatism. Early creep has been shown to affect the stress/strain relationship even over short time periods (see Fig. 3) with implications for suturing techniques. Further work will be needed to quantify this effect but it is probable that the creep behaviour of nylon sutures tends to correct for overtightening of sutures and helps to reduce the amount of suture-induced astigmatism.

\section{CONCLUSION}

A short pilot study into the mechanical properties of ophthalmic sutures has been completed. The results show that the Alcon 10/0 monofilament nylon suture is a mechanically consistent material in terms of its stress/strain relationship and that its creep deformity is likely to be an advantage. The suture itself seems not to contribute to variations in post-operative astigmatism.

The authors would like to thank Mr McNally of University of Northumbria Physics Department for his help in setting up the apparatus and Dr C. Armstrong and Professor K. Boddy for their encouragement throughout.

Key words: Cataract surgery, Mechanical properties. Ophthalmic sutures, Suture-induced astigmatism.

\section{REFERENCES}

1. Gimbel HV, Raanan, MG, DeLuca M. Effect of suture material on postoperative astigmatism. J Cataract Refract Surg 1992; 18:42-50.

2. Maloney WF, Grindle L, Sanders D, Pearcy D. Astigmatism control for the cataract surgeon: a comprehensive review of surgically tailored astigmatism reduction (STAR). J Cataract Refract Surg 1990;15:45-54.

3. Cory CC. Prevention and control of postimplantation astigmatism. J Cataract Refract Surg 1990;15:58-60).

4. Timoshemko S, MacCullough GH. Elements of strength of material. 3rd ed. New York: Van Nostrand, 1949:5-9.

5. Benham PP, Crawford RJ. Mechanics of engineering materials. 1st ed. Harlow, Essex: Longman, 1987:585-8.

6. Kronenthal R. Intraocular degradation of non-absorbable sutures. Am J Intraocular Implant Soc 1977;3:222-8.

7. Hutz W. Ullerich K. Microsurgical suture material. In: Draeger $\mathbf{J}$ (ed) Ophthalmic microsurgery: instrumentation, microscopes and technique. Basel: Karger, 1987:136-41. 